\title{
PAKISTAN AUF DEM WEGE ZU EINER NEUEN VERFASSUNG
}

\author{
Von Helmut R. Külz
}

General Jajah Khan, Pakistans „President and Chief Martial Law Administrator“, macht ernst mit seiner Zusage, so schnell wie möglich bürgerlich-demokratische Verhältnisse wiederherzustellen. Bereits am Tage nach seiner Machtübernahme in seiner ersten Botschaft an die Nation vom 26. März 1969 hatte er sich dafür verbürgt „to strive to restore democratic institutions in the country", wenn es auch zunächst noch verfrüht sei, für Wahlen einen Termin zu bestimmen. Auch Anfang Mai 1969, nach einer ersten Erkundungsrunde bei den maßgebenden Vertretern der verschiedenen politischen Kräfte des Landes, schien es ihm vorerst noch nicht möglich, die versprochenen Wahlen zwecks Rückkehr zu verfassungsmäßigen $\mathrm{Zu}$ ständen anzusetzen. Die Kluft zwischen den verschiedenen Anschauungen der verschiedenen Gruppen des Landes und insbesondere zwischen Ost- und West-Pakistan schien ihm dafür immer noch zu groß. Aber bereits nach kaum vier weiteren Monaten, unter dem 27. August 1969, erging die „Electoral Rolls Order 1969“ („President Order No 6 of $\left.1969^{“}\right)^{1}$, der erste bedeutende Schritt zur Vorbereitung von Neuwahlen.

In seiner zweiten großen Botschaft an die Nation vom 28. November $1969^{2}$ hielt der neue Präsident dann den Zeitpunkt für gekommen, einen genauen Zeitplan für den weiteren Ablauf der Entwicklung zu einer neuen Ordnung zu verkünden:

1. ab 1. Januar 1970 würde unter Fortfall aller kriegs- und ausnahmerechtlichen Einschränkungen wieder volle politische Betätigungsfreiheit gegeben sein;

2. bis zum 31. März 1970 werde er für die Wahlen zu einer verfassunggebenden Nationalversammlung eine erste Rahmenverordnung erlassen;

3. bis 1 . Juni 1970 werde die Fertigstellung der Wahllisten beendet sein und daraufhin durch eine Wahlkommission die Wahlkreiseinteilung festgelegt werden;

4. als Wahltermin werde der 5. Oktober 1970 bestimmt;

5. innerhalb von 120 Tagen nach Zusammentritt der Nationalversammlung werde die neue Verfassung verabschiedet sein müssen, sonst werden

6. die Nationalversammlung wieder aufgelöst sein und Neuwahlen zu einer anderen Nationalversammlung ausgeschrieben werden.

Die ersten drei Stufen dieses Planes sind programmgemäß und soweit ersichtlich ohne größere Schwierigkeiten und Verwicklungen beschritten und zurückgelegt worden. Bis zur nächsten Stufe, dem Wahltermin am 5. Oktober 19702a, werden noch vier Monate vergehen, in denen die Parteien und Gruppen des Landes sich endgültig formieren und ihre Kandidaten vorstellen werden; vier wichtige Monate, in denen die neue, im Werden begriffene Ordnung nochmals - oder besser schon im voraus - eine gewisse Bewährungsprobe abzulegen haben wird. Grund genug, die derzeitige Lage nach Zurücklegung der ersten drei Stufen des entwickelten Planes auch außerhalb des Landes etwas genauer zu untersuchen und einzuschätzen, insbesondere die am 29. März 1970 fristgemäß erlassene „Legal Framework Order 1970“ („Presidents Order No 2 of 1970“)

1 U. a. abgedruckt in The Pakistan Times v. 28. August 1969: nachfolgend als ERO angeführt.

2 Vgl. The Pakistan Times v. 29. November 1969.

2a Vgl. den Nachtrag des Verfassers am Ende des Beitrags.

3 Vgl. The Pakistan Tımes vom 30. März 1970, nachfolgend als LFrO angeführt. 
Zum Verständnis sind vorab kurz die politischen Hauptfragen zu umreißen, deren Lösung dem Nachfolger Ayub Khans aufgegeben war und ist. Sie sind an sich ganz klar und übersichtlich erkennbar und erschöpfend wie folgt aufzuzählen:

1. die Einführung des allgemeinen, gleichen und unmittelbaren Wahlrechts anstelle des bisherigen mittelbaren Wahlrechts durch sog. „basic democracies“ (etwa mit "demokratischen Grundbezirken" zu übersetzen),

2. die Einführung eines aus solchen allgemeinen und unmittelbaren Wahlen hervorgehenden demokratisch-parlamentarischen Systems, anstelle des bisherigen autoritären Präsidialsystems der alten pakistanischen Verfassung Ayub Khans von 1962,

3. die Einführung eines bundesstaatlichen Systems anstelle des Zentralismus der Verfassung von 1962, unter besonderer Beachtung

4. der unbedingten Gleichheit der parlamentarischen Sitzverteilung nach den abgegebenen Stimmen, anstelle des bisherigen, das volkreichere Ostpakistan benachteiligenden Grundsatzes der Gleichheit zwischen West- und Ostpakistan („parity“); sowie

5. der Aufteilung Westpakistans in einzelne stammesmäßig und sprachlich verschiedene Sonderprovinzen, anstelle des bisherigen Grundsatzes der Zusammenfassung Westpakistans zu einer einheitlichen Gebietskörperschaft („one unit"Grundsatz).

Alle diese 5 Punkte sind nun, dies muß als erstes hervorgehoben und im einzelnen noch kurz festgehalten werden, bereits im Vorgriff geregelt worden. Die verfassunggebende Versammlung hat also insoweit keinen Spielraum zu einer anderweitigen Entscheidung, was aber keine wirkliche Einschränkung einer freien Verfassungsschöpfung bedeutet. Denn im Grunde sind von den angeführten 5 Punkten die Hauptpunkte - zu 1., 2. und 4. - demokratische Selbstverständlichkeiten, die zu besonders klärungsbedürftigen Punkten nur dadurch geworden sind, $\mathrm{da}$ sie bisher anders, undemokratisch geregelt waren. Auch die von vornherein verordnete Festlegung auf eine ausgeprägte bundesstaatliche Ordnung und auf weitgehende Aufgliederung Westpakistans - zu 3. und 5. - ist keine eigentliche undemokratische, sondern aus den besonderen Verhältnissen des Landes fast zwangsläufig hervorgegangene Festlegung, in der geradezu die alleinige Gewähr für den Fortbestand des Landes überhaupt zu erblicken ist. Ohne Zusicherung einer ausgesprochenen bundesstaatlichen Ordnung und ohne Aufgliederung des bisher einheitlichen Westpakistan würde das Land mit ziemlicher Sicherheit schlechthin in verschiedene Teile auseinandergebrochen sein. Ungeachtet aller dieser notwendigen Vorentscheidungen wird die am 5. Oktober $1970 \mathrm{zu}$ wählende Nationalversammlung also keineswegs nur etwa über mehr oder weniger unwichtige und technische Ausführungsvorschriften zu befinden haben. Allenthalben sind vielmehr zu den erwähnten 5 Punkten noch wichtige grundsätzliche Sachentscheidungen zu treffen.

Was zunächst das Wahlrecht betrifft, so wird es allen 21jährigen männlichen und weiblichen Pakistanis zustehen, die dazu in genauen Wahllisten erfaßt worden sind. Sie sind seit Oktober 1969 in einem in der ERO genau geregelten Verfahren unter Aufsicht und Leitung eines Chief Election Commissioner von über 80000 Registration Officers, Supervisors und Enumerators sorgfältig aufgestellt worden, erstmalig anhand besonderer Wahl- und Haushaltslisten, und können unbedenklich als zuverlässige und ehrliche Grundlage für die Ausübung des Wahlrechts angesehen werden, anders als die früheren, ziemlich oberflächlich aufgestellten und gelegent- 
lich wohl auf manipulierten Wahllisten des basic-democracies-Systems. Die LFrO spricht in Sect. 6 Abs. 1 nochmals ausdrücklich den Grundsatz der Unmittelbarkeit der Wahl aus und setzt in Sect. 9 das passive Wahlalter auf 25 Jahre fest. Nicht wählbar aber sind - außer den Gruppen der Geisteskranken und zu längeren Freiheitsstrafen Verurteilten - die folgenden Gruppen, die ganz bewußt von der Beteiligung an der politischen Willensbildung ausgeschlossen bleiben sollen (Sect. 9, Abs. 1, Buchst. D-H):

Regierungsmitglieder aus der Zeit nach dem 1. August 1969; alle aktiven Berufsbeamten und Richter des Landes nebst ihren Ehegatten, sowie alle unehrenhaft entlassenen früheren Beamten; alle Personen, die unmittelbar oder mittelbar an einem Lieferungs- oder Dienstleistungsvertrag mit der Regierung beteiligt sind.

Hier wird das Bestreben der neuen Herren nach einem scharfen Trennungsstrich gegenüber der Vergangenheit, auch gegenüber der letzten Vergangenheit und nach Unterdrückung jeder Korruption sehr deutlich. Die Zahl der Abgeordneten der Nationalversammlung ist auf 313 festgelegt (Sect. 4 Abs. 1), wovon mindestens 13 weibliche Abgeordnete sein müssen. Diese werden, die einzige Ausnahme vom Grundsatz der Unmittelbarkeit der Wahl, von den zunächst allgemein gewählten 300 Mitgliedern - unter denen auch schon Frauen sein können - hinzugewählt, eine Vorschrift, die ähnlich auch schon in der alten Verfassung enthalten war. Das Verhältniswahlrecht ist nicht vorgesehen.

$\mathrm{Daß}$ die von der Nationalversammlung zu verabschiedende Verfassung den „fundamental principles of democracy" entsprechen muß, ist in Sect. 20, Abs. 3 ausgesprochen; die Grundsätze müssen weiterhin durch unmittelbare und freie Wahlen in bestimmten Zeitabständen gesichert sein, für die Bundes- wie für die Provinzialgesetzgebungen. Darüber hinaus müssen die Grundrechte des Bürgers in der Verfassung festgelegt, die Unabhängigkeit der Gerichte gewährleistet und diese auch zur Sicherung der Grundrechte anzurufen sein. Den demokratischen Grundprinzipien geht aber (Sect. 20, Abs. 2!) die Verpflichtung auf die Lehren des Islam vor (nochmals ausführlicher in Sect. 22 ausgesprochen), denen kein Gesetz widersprechen darf (so auch schon in der bisherigen Verfassung). Darüber, ob die neue demokratische Ordnung nach dem Muster der amerikanischen Verfassung als Präsidialdemokratie oder nach britischem System als eine parlamentarische Demokratie ausgestaltet werden soll, enthält die LFrO keine Festlegungen, wird also erst durch die Nationalversammlung entschieden werden.

Vor allem aber schreibt die LFrO in ganz bestimmter Weise eine neue bundesstaatliche Ordnung vor. Einerseits sollen zwar (Sect. 20, Abs. 1) die Provinzen und sonstigen Territorien des Landes so in einer Föderation vereinigt werden, daß die Unabhängigkeit, die territoriale Unversehrtheit und die nationale Solidarität Pakistans gesichert sind und daß die Einheit der Föderation in keiner Weise gefährdet werden kann. Andererseits sollen aber (Sect. 20, Abs. 4) alle öffentlichen Gewalten einschließlich der gesetzgebenden, der verwaltenden und der Finanzgewalt so zwischen der Bundesregierung und den Provinzen verteilt werden, daß diese eine möglichst starke Selbständigkeit („maximum autonomy“) in Gesetzgebung, Verwaltung und Finanzen haben, wiederum aber die Bundesregierung soviel Gewalt behalten soll, daß sie ihre Verantwortlichkeiten in den äußeren und inneren Angelegenheiten des Landes erfüllen sowie seine Unabhängigkeit und territoriale Unversehrbarkeit erhalten kann. Als weiterer Grundsatz ist dem Verfassungsgeber auferlegt (Sect. 20, Abs. 5), daß er innerhalb einer bestimmten Frist durch geeignete 
Gesetze und andere Maßnahmen die Beseitigung aller wirtschaftlichen und sonsti gen Ungleichheiten zwischen den verschiedenen Provinzen und Teilen des Landes vorsehen soll. Ob die Provinzen zu Staaten erhoben werden sollen, ist offengelassen; auch ist nicht etwa zwingend vorgeschrieben, daß zur Vertretung der Provinzen bei der Bundesgesetzgebung eine zweite Kammer nach Art eines Bundesrates geschaffen werden muß.

Als nächster Punkt der neuen Ordnung hatten wir den an die Stelle des bisherigen Gleichheitsgrundsatzes zwischen West- und Ostpakistan tretenden Grundsatz der Beteiligung aller Landesteile nach ihren Bevölkerungszahlen („population basis“, Sect. 4, Abs. 2 LFrO) festgehalten. Wieso man zunächst in der Verfassung von 1962 West- und Ostpakistan - ungeachtet ihrer verschiedenen Einwohnerzahlen je zur Hälfte, also nur im gleichen Maße, in Wirklichkeit also ungleich, am Parlament hatte teilnehmen lassen, ist hier nicht weiter zu erörtern; es mag dafür einige scheinbar gute Gründe gegeben haben. Jedenfalls war das nach seiner Bevölkerung und auch nach seiner wirtschaftlichen Betätigung, seinem Beitrag zum Sozialprodukt des Landes deutlich stärkere Ostpakistan auf die Dauer nicht mehr geneigt, diese seine Benachteiligung hinzunehmen, eine der Hauptursachen der blutigen Wirren zu Beginn des vorigen Jahres. So war es von Anfang an fast selbstverständlich, daß der sog. Gleichheitsgrundsatz aufgegeben und durch den bevölkerungsmäßig zu berechnenden Anteil an der Nationalversammlung ersetzt wurde. Die LFrO hat sich nicht darauf beschränkt, diesen Grundsatz in Sect. 4, Abs. 2 als solchen zu verkünden, sondern in einer Anlage (Schedule I) zur besseren Sicherheit und Veranschaulichung sogleich die ziffernmäßige Zusammensetzung ausgerechnet und festgelegt, und zwar auf der Grundlage des letzten Volkszählungsergebnisses von 1961. Da seither die Bevölkerung Ostpakistans stärker zugenommen haben dürfte als die Westpakistans, liegt darin noch immer eine gewisse Bevorzugung des Westens, die man aber wohl mangels anderer zuverlässiger Berechnungsunterlagen hingenommen hat. Auch so ist jetzt das Úbergewicht Ostpakistans deutlich erkennbar geworden. Die 300 aus allgemeinen Wahlen hervorgegangenen Abgeordnetensitze (ohne die 13 Frauensitze) verteilen sich nach Schedule I der LFrO auf die neuen 5 Provinzen folgendermaßen:

$\begin{array}{lr}\text { Ost-Pakistan (mit Hauptstadt Dacca) } & 162 \text { Sitze } \\ \text { Punjab (mit Hauptstadt Lahore) } & 82 \text { Sitze } \\ \text { Sind (mit Hauptstadt Karachi) } & 27 \text { Sitze } \\ \text { Baluchistan (mit Hauptstadt Quetta) } & 4 \text { Sitze } \\ \text { Nordwest-Grenzprovinz (mit Hauptstadt Peshawar) } & 18 \text { Sitze } \\ \text { Staatsgebiete unter der Zentralverwaltung } & 7 \text { Sitze } \\ \text { insgesamt } & 300 \text { Sitze }\end{array}$

Umstrittener, aber schließlich auch schon vorab festgelegt war endlich der letzte der 5 Hauptpunkte der neuen Ordnung, die Aufgliederung des bisher als „one unit" zusammengefaßten Westpakistan in vier vorstehend schon ersichtlich gemachte Teilprovinzen, ohne weitere obere Zusammenfassung. Auch hier mag es für das bisherige System der "one unit" manche guten Gründe gegeben haben, die aber der stärkeren Ausprägung und Entwicklung eines bestimmten Regionalismus und Föderalismus auch im westlichen Landesteil auf die Dauer doch nicht standhalten konnten. Schon Anfang 1970, vor der LFrO, war daher die West Pakistan Dissolution Order (Presidents Order No 1 of 1970) ergangen, die wir in der LFrO bestätigt und weiter ausgestaltet finden. Die 4 neuen Westprovinzen 
sind durchweg, wie schon angedeutet, stammesmäßig und sprachlich oder dialektmäßig bestimmt, wobei nur anzumerken ist, daß sie nach wie vor nicht nur geographisch, sondern auch durch das fast überall gesprochene und verstandene Urdu - weiterhin neben dem ostpakistanischen Bengali die Landessprache Pakistans - verbunden bleiben und nicht etwa den stammes-, glaubens- und sprachenmäßig so unselig verfeindeten indischen Staaten und Provinzen zu vergleichen sein werden. Eine Auf- oder Untergliederung des einheitlich bengalischen Ostpakistans war demgegenüber nie ernstlich vorgeschlagen worden; die offenbare Ungleichheit der neuen 5 Provinzen hat man wohl trotz der Probleme, die sich daraus ergeben können, als nicht entscheidend in Kauf genommen.

Úber die behandelten 5 Hauptpunkte hinaus enthält aber die LFrO noch einige andere sachliche und formelle Regelungen im Sinne des von Jajah Khan im November 1969 verkündeten Zeitplanes, und auch hierauf ist zum richtigen Verständnis der kommenden Verfassungsgestaltung noch kurz einzugehen. Es lassen sich der besseren Übersichtlichkeit halber wiederum die folgenden 4 Punkte herausstellen:

a) Die Nationalversammlung soll zunächst innerhalb von 120 Tagen nur die Verfassung beraten und verabschieden, also keinerlei sonstige Gesetzgebungsarbeit übernehmen. (Sect. 26, Abs. 1). Nach Inkrafttreten der neuen Verfassung und entsprechender Regierungsbildung soll sie aber ohne Neuwahlen weiterhin als erstes Parlament fungieren (Sect. 23).

b) Der Nationalversammlung ist in einem Anhang III (Schedule III) der LFrO auch schon eine genaue, bis ins einzelne gehende Geschäftsordnung auf den Weg gegeben worden;

c) Der Präsident hat sich selbst ausdrücklich jede Änderung der LFrO vorbehalten (Sect. 27), auch die Einberufung der ersten Sitzung der Nationalversammlung (Sect. 14) und die förmliche Bestätigung der Verfassung (Sect. 25).

d) Nach den Wahlen zur Nationalversammlung sollen alsbald auch Provinzialwahlen zu den 5 neuen Provinzialversammlungen stattfinden (Sect. 5 und 13).

Die verhältnismäßig kurze, bei Vermeidung von Auflösung und Neuwahlen angeordnete Fristsetzung für die Verabschiedung der neuen Verfassung ist ein Novum der Verfassungsschöpfung. Sie wird mit der gewiß zu bejahenden Dringlichkeit eines neuen Verfassungswerks sowie damit erklärt und gerechtfertigt, daß nach der bereits vorliegenden Festlegung der erwähnten 5 Hauptpunkte der neuen Ordnung der noch zu ergehende restliche Verfassungsstoff bei aller Gewichtigkeit nicht mehr so unermeßlich sei, daß er in 4 Monaten nicht zu bewältigen sein sollte. Daher auch die der Nationalversammlung zunächst auferlegte Beschränkung auf ihre Verfassungsaufgaben. Im übrigen ist klar, daß die 120-Tagesfrist einen gewissen heilsamen Druck auf die Nationalversammlung ausüben soll, damit sie sich nicht zerredet und tatsächlich zu einem positiven Ergebnis kommt, nur zu verständlich nach den Wirren um den Jahreswechsel 1968/69 und den langen, erfolglosen Versuchen Ayub Khans, auf einer Roundtable-Konferenz mit den Führern des Landes eine neue Verfassung gütlich abzusprechen. Die Drohung mit der sofortigen Auflösung und mit Neuwahlen bei erfolglosem Fristablauf wird auch, wie man annehmen darf, etwaigen verantwortungslosen Obstruktionsbestrebungen in der Versammlung durchaus entgegenwirken, da sie sich bei Neuwahlen kaum auszahlen werden. Wie man hört, will man der Versammlung auch - etwa nach Art des Entwurfs von Herrenchiemsee für das Grundgesetz einen ersten Entwurf einer Verfassung ausgearbeitet als Beratungsgrundlage zu- 
leiten. Ungewöhnlich mag es uns auch erscheinen, daß die Nationalversammlung gegebenenfalls nach Inkrafttreten der Verfassung ohne Neuwahlen als Parlament weiterbestehen soll. Auch dies ist indessen nach den besonderen Verhältnissen des Landes nicht unverständlich; die Kosten, Umständlichkeiten und auch Erregungen zweier schnell aufeinander folgender Wahlen hat Präsident Jajah Khan seinem Volke sicherlich aus gutem Grunde nicht zumuten wollen.

Auch die schon vorliegende Geschäftsordnung der Nationalversammlung dient vorwiegend der Sicherung der zügigen Verabschiedung einer neuen Verfassung. Täglich 9 Uhr früh (!) sollen in der Regel ihre Beratungen beginnen (Sect. 10). Dem Präsidenten der Versammlung (Sprecher) sind weitgehende Befugnisse gegen Mißbrauch der Redefreiheit in der Versammlung gegeben. So sind jederzeit Redezeitbegrenzungen nach Sect. 16 möglich, keine unsachlichen oder ermüdenden Wiederholungen (,irrelevant or tedious repetitions") erlaubt (Sect. 22) und vielfach sonstige Sicherungen für einen sachlichen und erfolgreichen Ablauf der Beratungen eingebaut.

Als Sicherheitsventile gegen unübersehbare Entwicklungen sind die von Präsident Jajah Khan sich selbst vorbehaltenen Sonderrechte zu verstehen, die schon bei der Einberufung der Versammlung bedeutsam werden können. Der Vorbehalt einer Änderung der LFrO mag besonders dann aktuell werden, wenn es etwa um die Uberschreitung der 120-Tagesfrist für die Beendigung des Verfassungswerks gehen sollte. Hier hätte die Versammlung nicht etwa die Befugnis, sich selbst ihr Mandat zu verlängern. Das wichtigste Vorrecht des Präsidenten ist die Bestätigung der verabschiedeten Verfassung; der Gedanke, sie etwa nach einem Volksentscheid zu entwerfen, hat Jajah Khan ausdrücklich abgelehnt.

Die in der LFrO schließlich auch noch bis spätestens 22. Oktober 1970 vorgeschriebenen Provinzialwahlen sind als weitere Unterstreichung der künftigen Bundesstaatlichkeit des Landes gedacht. Auch hier hat man zur Abkürzung des Verfahrens, und um von vornherein unfruchtbare Auseinandersetzungen abzuschneiden, sogleich die Größe der Provinzialversammlungen bestimmt; in Ostpakistan wird sie aus 310 Abgeordneten bestehen, also fast so groß wie die Nationalversammlung sein; die anderen Provinzialversammlungen sind erheblich kleiner (Punjab 186, Sind 62, Baluchistan 21 und NW-Grenzprovinz 42); wichtig ist dabei noch, daß nach Sect. 26, Abs. 2 die Provinzialversammlungen erst nach Verabschiedung der neuen Gesamtverfassung einberufen werden dürfen, ein durchaus folgerichtiger und sachlich gerechtfertigter Zeitablauf, wenn er auch den Provinzialversammlungen für die Zwischenzeit von ihrer Wahl bis etwa Ende des Jahres eine gewisse Selbstentsagung zumutet und sich vielleicht Widerstände dagegen ergeben - vor allem im unruhigen bengalischen Osten - daß sie so monatelang untätig Gewehr bei Fuß stehen sollen. Rahmenvorschriften für die Provinzialverfassungen sind vorerst der LFrO noch nicht gegeben worden; vor Verabschiedung der Gesamtverfassung würden sie auch wenig Sinn haben.

Der Plan macht dem Präsidenten Jajah Khan und seinen Beratern alle Ehre; man darf wohl annehmen, daß bei der Anlage und Ausarbeitung des Planes vor allem der erfahrene Justiz- und Verfassungsminister A. R. Cornelius mitgewirkt hat, ein ehemaliger Chief Justice (Präsident des Obersten Gerichtshofs) des Landes. Der glücklichen und erfolgreichen Durchführung des Planes bis zur Verabschiedung einer neuen Landes- und entsprechender Provinzialverfassung ist aller Erfolg zu wünschen. Pakistan würde damit nach verhältnismäßig kurzem Ausnahmezustand wieder $\mathrm{zu}$ gesicherten und geordneten Verhältnissen kommen und in 
der Lage sein, sich auch wieder seinen vor 1968 schon so hoffnungsvoll begonnenen wirtschaftlichen Entwicklungs- und Erschließungsaufgaben zu widmen. Und manchem europäischen und südamerikanischen Militärregime würde Pakistan dann als Vorbild dienen können.

\section{Nachtrag:}

Nach Abschluß der vorstehenden Arbeit (Anfang Juni 1970) hat sich bezüglich des Wahltermins (5. Oktober 1970) eine neue Sachlage ergeben. Er mußte durch einen Mitte August des Jahres verkündeten Erlaß des Präsidenten um zwei Monate bis Anfang Dezember des Jahres herausgeschoben werden, da katastrophale Überschwemmungen in Ostpakistan dort einen geregelten Wahlkampf und sonstigen Wahlablauf zunächst nicht mehr möglich erscheinen ließen. Es werden also bis zur Wahl voraussichtlich noch etwa weitere vier Monate vergehen. 